\title{
Un exemple d'inversion victimaire : l'accusation de meurtre rituel et ses formes dérivées
}

A case of victim inversion: The accusation of ritual murder and its derived forms

\section{Pierre-André Taguieff}

\section{(2) OpenEdition}

\section{Journals}

\section{Édition électronique}

URL : http://journals.openedition.org/aad/3500

DOI : $10.4000 /$ aad. 3500

ISSN : 1565-8961

Éditeur

Université de Tel-Aviv

\section{Référence électronique}

Pierre-André Taguieff, « Un exemple d'inversion victimaire : l'accusation de meurtre rituel et ses formes dérivées », Argumentation et Analyse du Discours [En ligne], 23 | 2019, mis en ligne le 18 octobre 2019, consulté le 28 novembre 2019. URL : http://journals.openedition.org/aad/3500 ; DOI : 10.4000/ aad.3500

Ce document a été généré automatiquement le 28 novembre 2019.

\section{(i) (-)}

Argumentation \& analyse du discours est mis à disposition selon les termes de la licence Creative Commons Attribution - Pas d'Utilisation Commerciale - Pas de Modification 4.0 International. 


\title{
Un exemple d'inversion victimaire : l'accusation de meurtre rituel et ses formes dérivées
}

\author{
A case of victim inversion: The accusation of ritual murder and its derived forms
}

\author{
Pierre-André Taguieff
}

1 La question abordée dans cet article peut paraître hautement déprimante : l'accusation de «meurtre rituel » à l'encontre des Juifs, sous ses formes directes ou dérivées. Avec cette accusation folle, impliquant une croyance totalement dénuée de base empirique, la criminalisation des Juifs atteint des sommets. S'ils se réduisaient à de simples fictions témoignant de la force de l'imagination humaine, les récits de «meurtre rituel », qui présentent les Juifs comme les "enfants", les disciples ou les agents du diable " meurtrier dès le commencement » selon l'évangile de Jean $(8,44)$-, relèveraient de la littérature d'épouvante et resteraient confinés dans un sous-genre du fantastique ${ }^{1}$. Il s'agit bien de légendes ou de récits de facture mythique. Mais ils se traduisent par des mobilisations politiques et des violences, quant à elles réellement meurtrières. Car ces récits de meurtres sont des objets de croyance qui entretiennent et stimulent des passions négatives, parmi lesquelles dominent la peur, la haine et le ressentiment. Ces passions sont fixées sur la figure essentialisée et criminalisée d'un peuple tout entier: les Juifs. Un peuple imaginé comme un peuple formé d'assassins potentiels. La haine des Juifs n'a certainement rien inventé de pire. Et pourtant elle s'est montrée fort inventive au cours de l'Histoire - certes dans les détails sordides plutôt que dans les grands récits accusatoires, dont le nombre est restreint.

2 De l'accusation de meurtre rituel, Jules Isaac écrivait en 1950 : «Il ne s'agit que d'une fable. Mais d'une fable calomnieuse, meurtrière, et qui a fait couler des flots de sang juif innocent. D'une fable qui a la vie dure». Et d'ajouter, mettant en garde ses contemporains contre la tentation de sous-estimer la puissance de séduction de cette fable criminalisante et criminogène : "Ne la repoussons pas d'un simple haussement d'épaule. Dans le Proche-Orient, croyance vivace, fortement enracinée dans la mentalité populaire, elle court encore les rues» (Isaac 1950: 1). Non seulement 
l'accusation mensongère, dans sa forme traditionnelle, est loin d'avoir disparu du champ des croyances, mais, depuis la création de l'État d'Israël et sous des formes dérivées, prenant pour cibles l'État juif et le "sionisme », elle joue un rôle croissant dans le nouveau discours de propagande antijuif. Ceux qui transmettent et font circuler cette accusation ne visent pas à alimenter le mépris à l'égard des Juifs et ainsi à justifier des modes d'exclusion ou des formes de discrimination. Il ne s'agit pas ici de "l'enseignement du mépris» analysé naguère par Jules Isaac (1962), mais d'un enseignement de la haine et de la peur qui, destiné à susciter de l'indignation, prépare le passage au «tout est permis » contre les Juifs, et ainsi justifie les actions les plus violentes contre eux.

Dans ce cas de figure, répétons-le, la diffamation d'un peuple tout entier a atteint des sommets. Elle consiste à imputer aux Juifs, comme un attribut essentiel, des pulsions sanguinaires et plus particulièrement homicides traduites par des pratiques rituelles d'ordre religieux, supposées consignées dans le Talmud - affirmation fausse, mais objet de croyance résistant aux mises au point érudites ${ }^{2}$. Dans les écrits antisémites modernes dénonçant la pratique du meurtre rituel chez les Juifs, celle-ci est régulièrement rapportée au Talmud ${ }^{3}$. Début janvier 1897, le journal La Croix assurait à ses lecteurs que " le Talmud leur permet de nous voler et de nous tuer" ». " Le Talmud est à la base de tous les méfaits des Juifs ", lisait-on le 20 juin 1940 dans Der Stürmer, fondé et dirigé par Julius Streicher, l'un des "spécialistes » nazis du meurtre rituel et du complot juif mondial. En décembre 1938, dans Le Pilori, revue raciste et antijuive explicitement pro-hitlérienne, un article d'une extrême virulence portait sur les Juifs, caractérisés comme les représentants d'une "race haineuse, sadique et criminelle » dont les traits seraient dévoilés dans le Talmud :

Non, le Juif n'est pas un homme comme les autres, pas plus que le loup n'est comparable aux autres races de chiens dont il est l'ennemi irréconciliable. [...] De toutes les lois qui régirent les nations, celle des Juifs fut la plus méprisable, la plus barbare, la moins digne d'un peuple policé. [...] C'est ce groupe-là qui a conçu, contre tous les autres peuples étrangers à sa race, une haine dont rien ne peut donner une idée sinon la lecture du Talmud (Le Français 1938 :9).

4 La lecture de ces idéologues et propagandistes antijuifs nous fait pénétrer dans un univers imaginaire cauchemardesque, peuplé d'êtres hideux, cruels et sanguinaires des monstres, mi-hommes mi-bêtes, et des démons à face semi-humaine. La bestialisation va ici de pair avec la démonisation.

5 Il convient donc d'étudier la formation et l'évolution du mythe du "meurtre rituel », matrice et véhicule du stéréotype du Juif cruel et sanguinaire, à travers ses trois grands moments historiques : antijudaïsme (antique et chrétien médiéval), antisémitisme (ou judéophobie moderne), antisionisme. Il s'agit avant tout de formuler ou de reformuler un certain nombre de problèmes suscités par l'analyse des formes historiques prises par l'hostilité à l'égard des Juifs. Dans la version du récit fixée entre le le douzième et le quatorzième siècle, les Juifs sont accusés de pratiquer des sacrifices humains suivis d'actes de cannibalisme, dont les victimes seraient des non-Juifs, en particulier des enfants. Or, l'on sait, comme le rappelle le récit du sacrifice d'Isaac dans la Bible ${ }^{5}$, que les sacrifices humains sont explicitement interdits par la loi juive ${ }^{6}$.

Dans les affaires de meurtre rituel, on accuse pourtant les Juifs de consommer du sang humain, ce qui est faire preuve d'ignorance volontaire de l'interdit biblique portant sur la consommation $\mathrm{du} \mathrm{sang}^{7}$. Point de preuves empiriques, mais des aveux extorqués sous la torture dans le cadre de procès organisés comme des spectacles édifiants (Kieval 
1994 : 1091-1105 ; Zimra $2010: 43-56)$ et une multitude de rumeurs qui se transforment en légendes populaires, lesquelles, par l'effet du biais de confirmation d'hypothèse ${ }^{8}$, renforcent les convictions antijuives déjà là. C'est mettre en circulation l'image du Juif cruel, sanguinaire et cannibale, qui, dans la littérature antijuive, donnera lieu au fantasme du « vampirisme juif », du Juif « suceur de sang ${ }^{9}$ ». C'est là aussi construire les non-Juifs - les chrétiens, mais aussi les musulmans -, et en particulier les enfants, comme formant la catégorie des victimes potentielles des Juifs.

7 Nous sommes en présence d'un ensemble de rumeurs et de légendes fabriquées et instrumentalisées, qui ont fini par donner naissance à un grand récit d'accusation, fonctionnant comme un mythe mobilisateur. Au Moyen Âge, le cadre théologicoreligieux des accusations de meurtre rituel est constitué par la démonologie : les Juifs sont perçus comme incarnant les forces des ténèbres et ainsi construits comme incarnant l'ennemi ${ }^{10}$. L'historien Joshua Trachtenberg rappelle que la chrétienté médiévale percevait le Juif comme «sorcier, meurtrier, cannibale, empoisonneur, blasphémateur, disciple du diable» (Trachtenberg 1983 [1943]). C'est dans ce cadre culturel que les Juifs ont commencé à incarner le mal absolu : meurtriers du Christ, représentants de l'Antéchrist - voire l'Antéchrist en personne - et agents zélés de Satan. La croyance à une alliance entre le diable et les Juifs, supposés suivre les préceptes secrets et criminels du Talmud, fournit un modèle interprétatif des accusations les plus chimériques, qui circulent sous la forme de rumeurs, lesquelles alimentent la judéophobie populaire (Berger 1997 ; Chazan 1997), une judéophobie qui prend la forme d'une démonomanie culturellement transmissible. L'image des Juifs est celle de créatures de Satan. Les prétendus «meurtriers du Christ » sont dès lors aussi perçus comme des assassins de chrétiens. La légende des Juifs empoisonneurs, qui circulait au Moyen Âge, sera reprise au $16^{\mathrm{e}}$ siècle par Martin Luther, qui affirmait que «si [les Juifs] pouvaient nous tuer tous, ils le feraient volontiers, certes, et ils le feraient souvent, spécialement ceux qui exercent la médecine ${ }^{11} »$.

8 L'accusation d'empoisonnement n'a pas cessé d'être portée contre les Juifs, indépendamment de son cadre théologique, comme en témoigne l'affaire des " médecins empoisonneurs », faux complot juif fabriqué de toutes pièces par Staline en 1952-1953. On la retrouve dans l'antisionisme contemporain, par exemple après la mort naturelle de Yasser Arafat le 11 novembre 2004 à l'hôpital militaire de Percy, à Clamart: selon une rumeur lancée par la propagande palestinienne, le leader palestinien aurait été empoisonné par les "sionistes ${ }^{12}$ ». Le biais de confirmation d'hypothèse permet de comprendre l'acceptabilité de cette accusation : elle confirme les attentes de ceux qui croient que les Juifs empoisonnent leurs ennemis. Que nous restions sans voix face à ces accusations délirantes, ou que nous ne les prenions pas au sérieux, cela est parfaitement compréhensible. Il faut reconnaître, à la suite de George Steiner, que, " pour des esprits entraînés au raisonnement, à une vision empirique de la réalité, il est difficile de saisir la force de possession d'une doctrine, même, ou particulièrement, quand cette doctrine apparaît comme irrationnelle, étrangère à la preuve et au témoignage concret » (Steine 1987 : 27). C'est le cas avec les « libelles de sang ».

Quoi qu'il en soit, le phénomène est observable: s'est ainsi constitué un stock de stéréotypes négatifs dans lequel vont puiser ultérieurement les auteurs de pamphlets antijuifs de toutes obédiences, qu'ils soient chrétiens ou antichrétiens. Car la sécularisation des accusations contre les Juifs, à l'exception de celle de déicide, n'a 
nullement interrompu, à partir du $18^{\mathrm{e}}$ siècle, le processus de transmission de leurs formes religieuses. Dans le dernier tiers du $19^{\mathrm{e}}$ siècle, les textes antijuifs mêlaient des motifs antijuifs expressément chrétiens et d'autres issus de la judéophobie antichrétienne des Lumières ${ }^{13}$. En 1894, dans son livre sur l'antisémitisme, Bernard Lazare dressait ce constat: "On retrouve dans les livres antisémites toutes les assertions des pamphlets du Moyen Âge, que déjà le XVII ${ }^{e}$ siècle avait repris, assertions que corroborent encore des croyances populaires. Mais le préjugé le plus vivace, celui qui symbolise le mieux le séculaire combat du judaïsme contre le christianisme, c'est le préjugé du meurtre rituel. Le Juif a besoin de sang chrétien pour célébrer sa pâque, diton encore ». Puis le futur héros de l'affaire Dreyfus s'interrogeait : " Mais comment les Juifs, dont les livres mosaïstes protestent de l'horreur du sang, ont-ils eu à pâtir et pâtissent-ils encore d'une telle croyance ?» (Lazare 1894 : 351-2).

Tout se passe comme si l'opposition du judaïsme aux sacrifices humains, et en particulier aux sacrifices d'enfants, loin de calmer les passions antijuives, les avaient exacerbées. Poliakov a formulé l'hypothèse selon laquelle la haine antijuive proviendrait $\mathrm{du}$ scandale provoqué par l'opposition du judaïsme aux sacrifices d'enfants. C'est précisément le respect de la vie humaine qui, chez les Juifs, ferait scandale, comme l'atteste ce passage de Tacite :

Ils ont un grand soin de l'accroissement de la population. Ils regardent comme un crime de tuer un seul des enfants qui naissent; ils croient immortelles les âmes de ceux qui meurent dans les combats ou les supplices; de là leur amour d'engendrer et leur mépris de la mort ${ }^{14}$.

11 Cette construction victimaire, pour être délirante, n’a cessé depuis le milieu du douzième siècle de déclencher des réactions violentes contre les Juifs - émeutes sanglantes et pogromes -, de légitimer des chasses aux Juifs ou de justifier des mesures d'expulsion. La désignation d'un groupe de coupables imaginaires constitue en ellemême un acte de stigmatisation globale dudit groupe, mais elle représente en même temps une incitation à la violence contre les membres du groupe stigmatisé et un puissant de mode de légitimation des actions violentes contre ces derniers. Il y a là l'illustration d'un processus ou d'un mécanisme victimaire qu'on peut présumer universel : l'auto-victimisation d'un groupe à travers des récits criminalisant un autre groupe, quant à lui réellement innocent des crimes qu'on lui impute (Taguieff 1983 : 133-156). L'inversion victimaire est le facteur déclenchant d'une forme de mobilisation sociale et politique dont on peut fournir de multiples exemples historiques. Ce mécanisme psychologique est au principe de la construction de l'ennemi absolu, celui qui incarne le Mal et contre lequel il faut lutter par tous les moyens (Cohn 1967 : 248-165).

12 Le concept de causalité diabolique, modèle élaboré par Léon Poliakov (2006), est ici fort éclairant. L'étude des "légendes de sang " conduit à distinguer, parmi les modes de diabolisation des Juifs, ceux qui sont centrés sur le projet d'une domination du monde et ceux qui tournent autour d'une pulsion meurtrière orientée vers les non-Juifs. Si les mythes complotistes se nourrissent du fantasme du Juif dominateur et conspirateur, visant la puissance mondiale, les mythes construits sur la base de l'accusation de meurtre rituel sont structurés par l'idée selon laquelle les Juifs poursuivent, en raison de leur nature - leurs instincts sanguinaires - ainsi que des enseignements - supposés de leur religion, l'élimination physique des non-Juifs. Le paradoxe est cependant à noter : dans cette configuration mythologique, les Juifs sont accusés de vouloir détruire le «matériel» humain dont ils ont besoin pour leurs rituels. Ce paradoxe s'ajoute à 
celui qui consiste, en même temps, à accuser les Juifs d'être mus par une volonté de dominer tous les peuples non juifs et à les accuser de vouloir exterminer ces derniers. Mais, tout comme l'inconscient, la pensée mythique ignore les contradictions formelles.

L'analyse des meurtres à caractère rituel imputés aux Juifs montre que l'inversion victimaire est à l'origine de la plupart des accusations contre les Juifs, aussi diverses soient-elles. Il s'agit d'un mécanisme consistant à substituer les victimes imaginaires aux victimes réelles et à transformer ces dernières en sujets criminels, en même temps que les vrais criminels sont présentés comme des victimes (réelles ou potentielles), des défenseurs ou des vengeurs des victimes. La souffrance des victimes paraît justifier tous les actes de vengeance. Elle donne le droit de riposter, de répliquer, elle fait de l'acte de vengeance l'objet d'un droit, conférant ainsi à l'esprit de vengeance des lettres de noblesse.

Encore faut-il que les victimes soient des victimes réelles, reconnues comme telles. La question devient dès lors celle de la reconnaissance des victimes, de leur labellisation. En prétendant venger les victimes appartenant à leur groupe, les pseudo-victimes font des victimes réelles parmi les Juifs, les victimes imaginaires s'en prennent violemment à leurs propres victimes. C'est la logique des pogromes, présupposant un besoin de réparation par la vengeance - ce qui n'implique nullement qu'on conçoive la vengeance comme un "phénomène universel ${ }^{15}$ ", quelque chose comme un besoin primordial ou une pulsion attribuée à la nature humaine (Lefranc 2016). Appliqué aux Juifs, le schème de l'inversion victimaire conduit à voir en eux l'incarnation d'une menace de mort pesant sur tout non-Juif. Si l'assassinat de la famille impériale russe, à la mi-juillet 1918, est un événement historique doté d'une exemplarité négative, c'est parce qu'il a été interprété aussitôt par les milieux antijuifs comme la preuve de l'existence d'un complot juif international et de la propension des Juifs à pratiquer des meurtres rituels. À leurs yeux, le pouvoir bolchevique étant l'expression de la puissance juive, les Juifs avaient décidé et commis ce meurtre hautement symbolique. Les pogromistes accusaient en quelque sorte les Juifs d'avoir organisé un pogrome anti-tsariste, c'est-àdire à la fois antichrétien et antirusse. Le schème de l'inversion victimaire s'est ainsi parfaitement appliqué. Pour ceux qui y croyaient, les deux grands mythes antijuifs trouvaient dans cet événement tragique une illustration historique dotée d'une valeur de preuve ${ }^{16}$. Or, cette croyance est récurrente.

Les Juifs sont ainsi construits comme un groupe de criminels par nature et culture, un peuple de «bouchers » meurtriers, incarnant un péril mortel contre lequel il importe au plus haut point de se protéger. Le désir de vengeance fonctionne pour ainsi dire à vide, comme s'il s'agissait de prévenir les meurtres commis par les Juifs. Cette passion est exclusive et impérative, comme le rappelait Hume : «Qui ne voit que la vengeance, par la seule force de la passion, peut être si avidement poursuivie qu'elle nous fait négliger toute considération de bien-être, d'intérêt et de sécurité et, comme pour certains animaux rancuniers, nous pousse à infuser nos âmes dans les blessures que nous infligeons à un ennemi?» (Hume 1751: 225). Par un apparent paradoxe, l'effervescence passionnelle, aussi meurtrière soit-elle, ne va pas sans un certain désintéressement.

Les enquêtes sur les affaires de meurtre rituel montrent que les "vengeurs" bricolaient un mode de légitimation de leurs actes de violence contre les Juifs sur la base de rumeurs locales et de légendes populaires - mettant ainsi entre parenthèse leurs passions (envie, jalousie, ressentiment, etc.) et leurs intérêts individuels (piller, 
violer, éliminer des concurrents, etc.). Il y a là une sorte de fanatisme élémentaire, qui se passe de justifications théologico-religieuses élaborées. Les croyances constitutives d'une religion populaire ne se modèlent pas nécessairement sur celles de l'orthodoxie religieuse. Dans les discours des auteurs de pogromes ou d'émeutes antijuives, l'entrecroisement des descriptions des prétendus assassinats commis par des Juifs, des violences réelles contre ces derniers et leurs supposés complices - en principe : tous les Juifs visibles - et des rationalisations mythiques des violences antijuives réelles est tel qu'il pose un problème d'interprétation, au cas par cas. C'est au nom des victimes qu'ils prétendaient représenter que les pogromistes assassinaient des Juifs, parce qu'ils croyaient ou paraissaient croire au mythe du Juif meurtier rituel. La question reste cependant sans réponse : les pogromistes croyaient-ils vraiment au mythe du meurtre rituel juif?

Évoquant en 1944 le mythe du complot juif mondial, Max Horkheimer et Theodor W. Adorno recourent au modèle de la projection pour rendre compte de l'adhésion à ce mythe : «Dans l'image du Juif que les racistes présentent au monde, ceux-ci expriment en fait leur propre nature. Ils sont avides de possession exclusive et d'un pouvoir illimité, à quelque prix que ce soit » (Horkheimer \& Adorno 1969 [1944] :78). Le même mécanisme fonctionne dans le cas de la croyance au meurtre rituel juif. Elle trouve son origine dans un puissant désir de tuer des Juifs. Ceux qui accusent les Juifs de pratiquer des meurtres rituels sont ceux qui en pratiquent ou rêvent d'en pratiquer contre les Juifs, sous la forme, elle-même ritualisée, d'émeutes sanglantes ou de pogroms. Sur ce plan psychologique, au sein de la communauté pogromiste, la différence entre les pogromistes actifs et les pogromistes potentiels tend à s'effacer. Horkheimer et Adorno notent en ce sens: "L'antisémitisme est un schéma bien rodé, un rituel de la civilisation et les pogromes sont de véritables meurtres rituels » (ibid. 180). C'est parce qu'ils sont porteurs de signes victimaires que les Juifs «semblent prédestinés pour attirer sur eux une telle projection " (ibid. 207). Ils ne sont donc pas arbitrairement choisis par leurs accusateurs ou leurs agresseurs. À cet égard, l'analyse développée par Adorno et Horkheimer rejoint celle de René Girard dans Le bouc émissaire (1982 : 28-29). Son principe est simple: dans des situations de crise ou d'anomie, on désigne un individu ou un groupe minoritaire comme coupable d'un crime qu'il n'a pas commis, la cible étant destinée à fixer l'hostilité.

Rappelons la description faite en 1899 par Émile Durkheim de ce mécanisme d'attribution causale: "Quand la société souffre, elle éprouve le besoin de trouver quelqu'un à qui elle puisse imputer son mal, sur qui elle se venge de ses déceptions; et ceux-là sont naturellement désignés pour ce rôle auxquels s'attache déjà quelque défaveur de l'opinion. Ce sont les parias qui servent de victimes expiatoires " (Durkheim 1975 [1899] : 252-254). On peut reconnaître à cette opération projective, impliquant une inversion victimaire, le statut d'un invariant anthropologique :

De tout temps l'assassin aveugle a vu dans la victime le persécuteur contre lequel il devait désespérément se défendre, et les empires les plus puissants ont toujours considéré le voisin plus faible comme une menace intolérable avant de l'assaillir. La rationalisation fut une ruse inévitable. Celui qui a été choisi comme ennemi est déjà perçu comme tel (Horkheimer \& Adorno 1969 [1944] : 195-196).

De son côté, rejoignant globalement les hypothèses ci-dessus exposées, le folkloriste étatsunien Alan Dundes, dans son étude sur le meurtre rituel, a proposé de l'aborder comme un mécanisme psychologique qu'il a baptisé l'« inversion projective » (projective inversion) et défini de la façon suivante: «L'inversion projective est un processus 
psychologique dans lequel A accuse B d'accomplir une action que lui-même, ou ellemême, souhaiterait accomplir " (Dundes 1991 : 28-29). C'est ainsi que, dans certains récits, le père est censé tuer ou vouloir tuer le fils, alors que c'est le fils qui désire tuer le père. Il en va de même pour les membres d'un groupe qui accusent les Juifs de meurtre rituel, alors que ce sont eux qui veulent tuer les Juifs. Le «tué par les Juifs » proféré par des accusateurs est le produit d'une inversion projective : il révèle chez les accusateurs un désir plus ou moins honteux de tuer des Juifs. Il en va de même pour la transformation du «Je vous hais » en "Vous me haïssez " (ibid. 355). Ce mécanisme constitue un mode de légitimation et de rationalisation de la violence exercée par le groupe accusateur contre le groupe accusé. Il remplit en outre une fonction d'incitation à l'agression et de mobilisation contre l'ennemi fantasmé.

On rencontre le mécanisme de l'inversion projective dans la vision qu'avait des Juifs le pape Grégoire le Grand (vers 540-604). On a de bonnes raisons, sur la question, de supposer sincère ce grand pape qui, «loin d'être un fanatique, s'est illustré par des qualités insignes, de générosité de cœur, d'élévation morale, d'équité, d'humanité ", comme le soulignait Jules Isaac (1956: 287, 230-235). Mais cet esprit modéré n'en réduisait pas moins les Juifs à un peuple "plus attaché à la terre qu'à la vérité » thème du "peuple charnel»-, ce qui le conduisait à voir en eux le peuple de «la Bête ", de "l'Antéchrist » et du " démon », un peuple animé d'une perversité satanique, d'une haine implacable contre Dieu et ses défenseurs, les chrétiens (ibid. 290-291). On trouve chez Grégoire $I^{\text {er }}$ les principales accusations constitutives de l'antijudaïsme chrétien, sans la virulence d'un Jean Chrysostome. Lisons quelques passages sur les Juifs extraits de l'un de ses principaux ouvrages rédigé peu après 579, les Moralia, les Morales sur Job :

Parce que les cœurs des Juifs sont vides de foi, ils sont soumis au diable [...] La Synagogue ne s'est pas contentée d'être réfractaire à la foi, elle l'a combattue par le glaive et a soulevé contre elle les horreurs d'une persécution sans merci [...] N’est-il pas exact de dire que la Bête a fait son repaire des cœurs des Juifs persécuteurs? [...] Plus l'Esprit Saint remplissait le monde, plus la haine perverse enchaînait les âmes juives [...] Leur aveuglement est allé jusqu'à la cruauté, et leur cruauté les a poussés à la persécution implacable ${ }^{17}$.

21 Jules Isaac a justement noté que «ces formules pourraient être inversées et mises au compte des chrétiens persécuteurs ${ }^{18} »$ (ibid. 290).

La justification des violences antijuives par des actes inhumains imputés aux Juifs peut apparaître hors de l'espace des écrits appelant à la haine envers les Juifs. Au $20^{\mathrm{e}}$ siècle, on observe une banalisation de la thèse selon laquelle les Juifs sont, par leurs comportements, la cause de la haine qui les vise. Dans son Histoire de la magie et de la démonologie, parue en 1926, le pasteur anglican Montague Summers (1880-1949) auteur d'ouvrages sur les sorcières et les vampires -, affirmait, comme l'a souligné justement Joshua Trachtenberg, que les Juifs avaient été persécutés en Moyen Âge «non pas tant en raison de l'observance des cérémonies hébraïques, comme on l'a souvent supposé et suggéré, qu'en raison des sombres et affreuses traditions de la magie hébraïque " (Trachtenberg 1926: 195). Et d'ajouter : "Les meurtres rituels étaient étroitement liés à ces actes de magie. [...] Dans de nombreux cas, les preuves sont évidentes du fait que le corps, et surtout le sang de la victime, était utilisé à des fins magiques ${ }^{19}$.»

23 Après la Deuxième Guerre mondiale et le grand massacre nazi des Juifs d'Europe, nombreux sont ceux qui ont cru que la page était tournée. Il n'en est rien. Le mythe du 
Juif meurtrier rituel hante toujours l'imaginaire social et se traduit par la circulation de stéréotypes criminalisants dans les nouveaux discours judéophobes prenant pour cibles privilégiées Israël, le «sionisme» et les «sionistes». La métamorphose des composantes du mythe est toujours en cours, faisant surgir un nouveau paysage antijuif international dont l'évolution est largement due à l'importance croissante des réseaux sociaux dans le processus de formation de l'opinion ainsi que dans la transmission des représentations et des croyances. La globalisation des accusations antijuives favorise autant la circulation à grande vitesse des rumeurs, des légendes et des "fake news " que leur adaptation à divers publics, selon les objectifs des propagandistes.

Il faut en effet distinguer le discours antijuif à usage interne, dans un espace national ou régional, et le discours antijuif d'exportation, fabriqué pour être idéologiquement acceptable par tel ou tel public visé. L'un des exemples les plus frappants des variations contemporaines du discours antijuif est offert par les stratégies d'accommodation que mettent en œuvre les propagandistes arabo-musulmans pour séduire et mobiliser le public occidental. Certains thèmes d'accusation contre les Juifs peuvent être exportés sous la condition d'être reformulés eu égard aux croyances en cours dans l'auditoire visé. Comme le note l'historien israélien Raphael Israeli, «l'outil le plus efficace dont disposent les antisémites arabes pour rallier les chrétiens est encore l'accusation de crime rituel, qui, sous sa forme la plus crue, accuse les Juifs de verser le sang chrétien à la veille de la Pâque juive, pour la fabrication de la matza (pain azyme)» (Israeli 2004 : 130). Mais l'historien ne manque pas d'ajouter que l'accusation " peut aussi prendre d'autres formes : les Juifs sont accusés d'empoisonner les puits, de diffuser la peste et, en termes plus modernes, d'utiliser de l'uranium appauvri contre les Palestiniens, de distribuer des bonbons empoisonnés ou d'injecter le virus du sida aux enfants palestiniens » (ibid.). Cette distinction entre les formes premières, «classiques » ou traditionnelles et les formes dérivées des récits accusatoires visant les Juifs (ou « les sionistes ", substitut lexical ordinaire) constitue un présupposé méthodologique de toute analyse rigoureuse de ce que j'ai appelé la «nouvelle judéophobie » (Taguieff $1989: 1-80$ et 2002).

La réinscription du thème d'accusation mythique dans le paysage antijuif contemporain est indissociable du processus de nazification des Juifs, en tant que "sionistes" fantasmés comme "racistes", "impérialistes» et "assassins ». La propagande antijuive contemporaine se présente à cet égard comme le produit d'un amalgame idéologique entre la nazification des Juifs-sionistes et la réactivation de la représentation du Juif " assoiffé de sang », cruel et sanguinaire - rejeton de l'imaginaire du meurtre rituel. Trois autres composantes du paysage antijuif désormais observable en marquent la nouveauté. Tout d'abord, l'islamisation croissante de la rhétorique judéophobe en même temps que l'intégration dans le discours islamiste d'accusations dont celle du meurtre rituel - empruntées à l'antijudaïsme chrétien européen (Wistrich 2002 : 11-15, 31-34; Israeli 2002 et 2012). Ensuite, le recyclage du discours antifasciste dans le cadre de la lutte contre Israël, «le sionisme» et "les sionistes", entités diabolisées de diverses manières. Enfin, la réactivation du stéréotype du «Juif riche », ploutocrate, capitaliste ou «banquier international », accusé de " tirer les ficelles » de la politique mondiale, stéréotype dont on observe la circulation dans les critiques radicales, d'extrême gauche ou d'extrême droite, de la mondialisation. Cette dénonciation hyperbolique de la mondialisation redonne vie au mythe répulsif, coloré de conspirationnisme, centré sur la figure de "Rothschild», incarnation de la puissance abusive de l'argent. Mais, dans la nouvelle propagande antijuive, Rothschild 
peut aussi se transformer en Shylock, comme lorsque le 15 mars 1997, Othman Abou Gharbiya, conseiller politique de Yasser Arafat, déclare à la radio: "Nous luttons et combattons contre un ennemi qui est Shylock. Nous devons savoir qu'il s'agit de Shylock $^{20}$ ». Le stéréotype de l'usurier juif cruel, avide et prédateur est au cœur de la représentation antijuive de l'ennemi, celle qu'on retrouve dans le discours " antisioniste » radical. C'est ainsi que le quotidien officiel de l'Autorité palestinienne, Al-Hayat al-Jadida, le 6 août 1997, criminalise l'« ennemi qui découvre ses crocs juifs aux quatre coins de la terre » (cité par Bensoussan 2004).

Ces représentations de l'ennemi juif, qui viennent de loin, sont donc réinvesties dans le discours «antisioniste» contemporain. L'ennemi désigné est présenté comme impitoyable, et sa cruauté est attestée par les récits de meurtres rituels dans lesquels les corps des jeunes victimes des Juifs sont souvent décrits comme "horriblement mutilés » (Favre-Vassas 1994 : 149-150). Les Juifs meurtriers rituels sont ainsi accusés de prendre une joie sadique à torturer leurs victimes sacrificielles, saignées et dépecées vivantes.

On observe le recyclage de cette thématique dans la propagande "antisioniste " contemporaine qui, relayée par les médias et les réseaux sociaux, constitue une vaste et puissante opération d'endoctrinement ${ }^{21}$. La doctrine enseignée et diffusée peut être baptisée simplement «antisionisme». Pour ses propagateurs, elle constitue une doctrine de salut : la voie de la rédemption se confond avec la destruction de «l'entité sioniste " et la création d'un "monde sans le sionisme» au terme d'un combat apocalyptique. Le 26 octobre 2005, le président iranien Mahmoud Ahmadinejad, dans un discours prononcé devant 4000 "étudiants " réunis en congrès à Téhéran sur le thème "Le monde sans le sionisme ", a lancé un appel à la destruction d'Israël :

La création du régime qui occupe Al-Qods [Jérusalem] a été une manœuvre significative du système globalement dominant et de la Globale Arrogance [l'Occident] contre le monde islamique. [...] Le Monde Oppresseur a créé le régime qui occupe Al-Qods pour qu'il soit la tête de pont de sa domination du monde islamique. [...] Aujourd'hui, la nation palestinienne combat le Monde Oppresseur pour l'Oumma [nation] islamique tout entière. [...] Notre cher Imam [Khomeiny] a ordonné que le régime qui occupe Al-Qods soit rayé de la surface de la terre ${ }^{22}$.

Cette propagande ne se réduit donc pas à une posture ou à une opinion politique. L'amalgame polémique sloganisé qu'elle fait circuler n'est autre que "sionistes = assassins ». Cet endoctrinement à visée planétaire à pour objectif de criminaliser les Israéliens et plus largement les « sionistes » sur la base d'une redéfinition polémique de l'identité « sioniste », l'extension de l'application de la catégorie impliquant d'inventer des formes subtiles de complicité avec les «sionistes assassins». Dans cette perspective, illustrant une forme de paranoïa tactico-stratégique, est « sioniste » tout Juif qui n'est pas explicitement anti-israélien et tout non-Juif qui n'est pas clairement propalestinien - ces non-Juifs «sionistes " prennent donc la relève de ceux que les antijuifs traitaient naguère d'«enjuivés». Il s'ensuit que le monde est peuplé d'innombrables "sionistes", Juifs et non-Juifs. Bref, une fois de plus, "ils sont partout », mais d'une façon nouvelle, car le " péril juif » est devenu le péril sioniste. Et, corrélativement, les prétendues victimes des « sionistes » se multiplient sans fin. Surgit ainsi un monde bariolé peuplé des victimes imaginaires de « sionistes » chimériques.

Dans une telle situation, l'enjeu politique peut se définir par les questions suivantes: pour les «antisionistes» de gauche et de droite, les «sionistes» ainsi redéfinis peuvent-ils incarner le nouvel ennemi commun? Les victimes fantasmatiques du diable 
«sioniste » vont-elles pouvoir et vouloir se fédérer, pratiquer l'union dans les luttes par-delà les ordinaires clivages idéologiques, ethniques et religieux ? Les extrémistes de tous bords trouveront-ils dans la cause antisioniste des raisons de se mobiliser ensemble? Les modérés de toutes obédiences en viendront-ils à communier avec les extrémistes dans la chasse aux "sionistes »? Le fait même que ces questions se posent aujourd'hui témoigne de l'inquiétude des esprits les plus lucides.

\section{BIBLIOGRAPHIE}

Bensoussan, Georges. 2004. « Antisémitisme et négationnisme dans le monde arabo-musulman : la dérive » (Editorial), Revue d'histoire de la Shoah 180

Benz, -Wolfgang. 2005 [2004]. Was ist Antisemitismus? (Munich : Beck)

Berger, David. [1997] 1986. « Anti-Semitism : An Overview », History and Hate: The Dimensions of Anti-Semitism (Philadelphia, New York \& Jérusalem : The Jewish Publication Society), 3-14

Chazan, Robert. 1986. « Medieval Anti-Semitism », David Berger (ed.). History and Hate : The Dimensions of Anti-Semitism (Philadelphia, New York \& Jérusalem : The Jewish Publication Society)

Chazan, Robert. 1997. Medieval Stereotypes and Modern Antisemitism (Berkeley \& Los Angeles : California U. P.)

Cohn., Norman. 1967. Histoire d'un mythe. La « conspiration » juive et les Protocoles des Sages de Sion, trad. Léon Poliakov (Paris : Gallimard)

Crapez, Marc. 1997. La Gauche réactionnaire. Mythes de la plèbe et de la race dans le sillage des Lumières, préface de P.-A. Taguieff (Paris : Berg International)

Darmon, Claire. 2004. «L'antisémitisme musulman : un danger très actuel », Revue d'histoire de la Shoah 180, 28-33, 42-47 (=trad. partielle de Wistrich 2002)

Desportes, Henri. 1889. Le mystère du Sang chez les Juifs de tous les temps, préface d'Édouard Drumont (Paris : Savine, « Bibliothèque antisémitique »)

Dundes, Alan. 1991. «The Ritual Murder or Blood Libel Legend : A Study of Anti-Semitic Victimization through Projective Inversion ", Dundes, Alan (ed.). 1992. The Blood Libel Legend : A Casebook in Anti-Semitic Folklore (Madison \& London : Wisconsin U. P.)

Durkheim, Émile. 1975 [1899]. « Antisémitisme et crise sociale », Textes, vol. II : Religion, morale, anomie, Victor Karady éd. (Paris : Minuit)

Elster, Jon. 1990. « Norms of Revenge », Ethics $100: 4,862-885$

Fabre-Vassas, Claudine. 1994. La bête singulière. Les juifs, les chrétiens et le cochon (Paris : Gallimard)

Faure, Marcel (éd.). 1999. Le sang au Moyen Âge (Montpellier : Cahiers du CRISMA 4)

Girard, René. 1982. Le bouc émissaire (Paris : Grasset) 
Horkheimer, Max \& Theodor W. Adorno. 1969 [1944] « Éléments de l'antisémitisme. Limites de la raison », La dialectique de la Raison. Fragments philosophiques, tr. fr. Éliane Kaufholz (Paris : Gallimard)

Hume, David. 1991 [1751]. Enquête sur les principes de la morale, trad. Philippe Baranger \& Philippe Saltel (Paris : Garnier-Flammarion)

Isaac, Jules. 1962. L'enseignement du mépris. Vérité historique et mythes théologiques (Paris : Fasquelle)

Isaac, Jules. 1956. Genèse de l'antisémitisme. Essai historique (Paris : Calmann-Lévy)

Iancu, Carol. 2010. « Les réactions des milieux chrétiens face à Jules Isaac », Revue d'Histoire de la Shoah 192, 157-190

Israeli, Raphael. 2002. Poison : Modern Manifestations of a Blood Libel (Oxford : Lanham \& New York : Lexington Books)

Israeli, Raphael. 2004. «L'antisémitisme travesti en antisionisme », (tr. fr. Jean-Pierre Ricard), Revue d'histoire de la Shoah, 109-171

Israeli, Raphael. 2012. Blood Libel and Its Derivatives : The Scourge of Anti-Semitism (New Brunswick \& London : Transaction Publishers)

Kieval, Hillel J. 1994. « Antisémitisme ou savoir social ? Sur la genèse du procès moderne pour meurtre rituel » (trad. Françoise Marin), Annales HSS 49 : 5, 1091-1105, https://www.persee.fr/ doc/ahess_0395-2649_1994_num_49_5_279313

Kriegel, Maurice. 1979. Les Juifs à la fin du Moyen Âge dans l'Europe méditerranéenne (Paris: Hachette) Laqueur, Walter. 1990 [1965]. Russia and Germany: A Century of Conflict (New Brunswick : Transaction Publishers)

Lazare, Bernard. 1894. L'antisémitisme, son histoire et ses causes (Paris : Chailley)

Lefranc, Sandrine. 2016. « La vengeance des victimes », https://halshs.archives-ouvertes.fr/ halshs-01524800/document

Le Français, François. 1938. « La race haineuse, sadique et criminelle », Le Pilori $1: 5$

Monniot, Albert. 1914. Le crime rituel chez les Juifs, préface d’Édouard Drumont (Paris : Téqui)

Normand, Linn. 2016. Demonization in International Politics : A Barrier to Peace in the IsraeliPalestinian Conflict (New York : Palgrave Macmillan)

Poliakov, Léon. 1955. Histoire de l'antisémitisme, vol. I : Du Christ aux Juifs de Cour (Paris : CalmannLévy)

Poliakov, Léon. 1965. « Le diable et les Juifs (La "diabolisation" des Juifs en Occident) », Max Milner (éd.), Entretiens sur l'homme et le diable (Paris \& La Haye : Mouton), 189-201

Léon Poliakov, « L'antisémitisme est-il un racisme? », Michel Wieviorka (éd.). 1993. Racisme et modernité (Paris : La Découverte)

Poliakov, Léon. 2006. La causalité diabolique [1980, 1985], préface de P.-A. Taguieff (Paris :

Mémorial de la Shoah \& Calmann-Lévy)

Reboul , Olivier. 1997. L'endoctrinement (Paris : PUF)

Reinach, Théodore. 1983 [1895]. Textes d'auteurs grecs et romains relatifs au judaïsme (Paris : Leroux) (réimpr., Hildesheim, Zurich \& New York : Olms) 
Ross, Lee, Mark R. Lepper \& Michael Hubbard. 1975. « Perseverance in Self-Perception and Social Perception : Biased Attributional Processes in the Debriefing Paradigm ", Journal of Personality and Social Psychology $32: 5,880-892$

Ross, Lee \& Mark R. Lepper. 1980. « The Perseverance of Beliefs : Empirical and Normative Considerations ", Shweder, Richard A. \& Donald W. Fiske (eds.). 1980. New Directions for Methodology of Behavioral Science : Faillible Judgment in Behavioral Research, 17-36 Roux, Jean-Paul. 1988. Le sang. Mythes, symboles et réalités (Paris : Fayard)

Shweder, Richard A. \& Donald W. Fiske (eds.). 1980. New Directions for Methodology of Behavioral Science : Faillible Judgment in Behavioral Research

Steiner, George. 1987. « La longue vie de la métaphore. Une approche de la Shoah », trad. Marie Moscovici, L'écrit du temps 14-15, 3-14

Slater, Wendy. 2007. The Many Deaths of Tsar Nicholas II : Relics, Remains and the Romanovs, (London \& New York : Routledge)

Strack, Hermann L.. 1892. Der Blutaberglaube in der Menschheit, Blutmorde und Blutritus (Munich : Beck)

Tacite. Histoires vol. V, § 5, Théodore Reinach. 1895. Textes d'auteurs grecs et romains relatifs au judaïsme (Paris : Leroux)

Taguieff, Pierre-André. 1983. « Sur une argumentation antijuive de base : l'auto-victimisation du narrateur ", Sens 7, 133-156

Taguieff, Pierre-André. 1989. « La nouvelle judéophobie. Antisionisme, antiracisme, antiimpérialisme », Les Temps modernes 520, 1-80

Taguieff, Pierre-André. 2006. L'imaginaire du complot mondial. Aspects d'un mythe (Paris : Fayard/ Mille et une Nuits)

Taguieff, Pieree-André. 2002. La nouvelle judéophobie (Paris : Fayard/ Mille et une nuits)

Tobias, Norman C. 2018. La conscience juive de l'Église. Jules Isaac et le concile Vatican II, trad. John E. Jackson, préface de Robert O. Paxton (Paris : Salvator)

Tokarska-Baki, Joanna. 2015 [2008] Légendes du sang. Pour une anthropologie de l'antisémitisme chrétien, tr. fr. Malgorzata Maliszewska (Paris : Albin Michel).

Trachtenberg, Joshua. 1926. The History of Witchcraft and Demonology (London : Kegan Paul, Trench, Trubner \& Co.)

Trachtenberg, Joshua. 1939. Jewish Magic and Superstition : A Study in Folk Religion (New York : Behrman's Jewish Book House)

Trachtenberg, Joshua. 1983 [1943]. The Devil and the Jews : The Medieval Conception of the Jew and Its Relation to Modern Antisemitism (New Haven : Yale U. P.) (sec. edition, preface Marc Saperstein (Philadelphia : The Jewish Publication Society of America)

Verdier, Raymond. 2004. « Une énergie vitale», R. Verdier (éd.). Vengeance. Le face-à-face victime/ agresseur (Paris : Autrement)

Wilton, Robert \& George Gustav Telberg. 1920, The Last Days of the Romanovs (New York : Doran)

Wistrich, Robert S.. 2002. Muslim Anti-Semitism : A Clear and Present Danger (New York: The American Jewish Committee) 
Zimra, Georges. 2010. « Le mystère du sang ", Topique 113, 43-56 ; https://www.cairn.info/revuetopique-2010-4-page-43.htm

\section{NOTES}

1. Indépendamment des figures ou des motifs qu'il sollicite (revenants, vampires, sorcières, loups-garous), le fantastique naît de la perception d'un événement étrange et inquiétant, qui paraît impliquer une rupture inexplicable de l'ordre reconnu, comme si le surnaturel faisait irruption dans la réalité pour la bouleverser et l'insécuriser. L'expérience du fantastique suppose le doute, qui plonge le sujet dans le domaine de l'incertitude et de l'ambiguïté, mais ouvre aussi au monstrueux et au terrifiant. Loin donc de sembler agrémenter, compléter ou parachever l'ordre naturel, comme le fait le merveilleux, le fantastique parait l'agresser, le contredire ou le transgresser, suscitant ainsi le trouble, voire l'effroi.

2. L'une des études historiques et critiques les plus savantes est celle de Hermann L. Strack, 1892.

3. Voir par exemple, en français : Desportes 1889, 9-37, Monniot 1914, 73-136.

4. « Le baptême d'un Juif », La Croix, 2 janvier 1897.

5. Gen., XXII, 1-14. Alors qu'Abraham était prêt à sacrifier son fils Isaac à Dieu, un ange lui enjoignit de ne lui faire aucun mal, exprimant ainsi la réprobation divine à l'égard des sacrifices humains.

6. Voir Lev., XVIII, 21 ; XX, 2.

7. Sur l'interdit du sang, voir Gen, IX, 4 ; Lev., III, 17 ; VII, 26-27 ; XVII, 12-14 ; Deut., XII, 12 ; XII, 23-25. Sur les représentations, les croyances et les interdits concernant le sang dans la sphère religieuse, voir Roux 1988, Faure 1999, V. Rousseau 2005, 5-130.

8. Le biais de confirmation est un biais cognitif qui nous incite à privilégier les informations allant dans notre sens et à écarter celles qui contredisent nos certitudes. Il a pour effet d'affermir les croyances, aussi fausses ou douteuses soient-elles, et, partant, d'empêcher toute recherche de la vérité. Voir Ross, Lepper \& Hubbard 1975., 880-892, Ross \& Lepper 1980, 17-36.

9. Voir les nombreux exemples textuels et iconographiques présentés dans le remarquable ouvrage de Joanna Tokarska-Baki. 2015 [2008].

10. Poliakov. 1955, vol. I , en partic. pp. 140-187 et 1965, 189-201, N. Cohn 1967 et 1982, Kriegel 1979, 37-38, Benz 2005, 65-77

11. Luther, cité par Trachtenberg. 1983, 99. Voir aussi Cohn 1967, 258.

12. La presse généraliste s'est fait l'écho de cette accusation d'assassinat. Voir par exemple « Les zones d'ombre autour de la mort de Yasser Arafat », 27 novembre 2012, https://www.lemonde.fr/ proche-orient/article/2012/11/27/les-zones-d-ombre-autour-de-la-mort-de-yasser-

arafat_1796344_3218.html; Armin Arefi, « Yasser Arafat a-t-il été assassiné ? », 14 novembre 2014, https://www.lepoint.fr/monde/yasser-arafat-a-t-il-ete-assassine-14-11-2014-1881234_24.php.

13. Sur le développement de "l'antisémitisme antichrétien » en France au 19e siècle, dans le sillage des écrits du baron d'Holbach et de Voltaire, voir Crapez 1997, 137-156.

14. Tacite. Histoires, V, § 5, Reinach $1895:$ 307-308. Voir Poliakov $1993: 84$ et $1994: 11-12$.

15. Elster $1990: 862$. Voir aussi Raymond Verdier 2004, 8-23.

16. L'un des premiers auteurs antisémites occidentaux à avoir accrédité cette interprétation de l'assassinat de la famille Romanov est le journaliste britannique Robert Wilton (1868-1925), correspondant en Russie du Times. Il l'expose dans le livre qu'il a co-signé avec le juriste George Gustav Telberg. 1920, intitulé The Last Days of the Romanovs. Sur les activités de Wilton, voir Laqueur 1965, 103, 323-324 ; Cohn 1967, 154-155 ; Slater 2007, 71-74..

17. Grégoire le Grand, Moralia ; passages cités par Isaac, op. cit., 290

18. Isaac, op. cit., 290 
19. Ce passage est cité et commenté par Trachtenberg 1939, 8 et 1983, 155. Voir aussi Poliakov $1955,296$.

20. Othman Abou Gharbiya, 15 mars 1997, cité par Bensoussan 2004, 14. Voir aussi Normand 2016,116 .

21. Sur la propagande et l'endoctrinement, voir Reboul 1997, 13-38.

22. Extraits de la version française intégrale du discours du président iranien rapporté par l'agence de presse ISNA, qui appartient au gouvernement iranien, le 26-10-2005. Voir Taguieff 2006, 169-178.

\section{RÉSUMÉS}

L'analyse du dispositif victimaire, proposé ici, s'appuie sur l'accusation de meurtre rituel portée contre les Juifs. Cette accusation rend possible la mise en place d'une construction victimaire des haïsseurs des Juifs, que ces derniers utilisent afin de justifier et de légitimer les mesures d'expulsion et d'extermination des Juifs depuis le douzième siècle. Sont analysés ici les ressorts de l'inversion victimaire qui s'est produite déjà à l'époque de l'antijudaïsme chrétien et qui ne cesse d'être rejouée dans les discours légitimant la violence contre les Juifs et aujourd'hui contre l'État hébreu. Ce phénomène obéit à un mécanisme rodé depuis des siècles; on l'observe aujourd'hui dans certains discours médiatiques et sur les réseaux sociaux. Il consiste dans la mise en scène de l'autovictimisation d'un groupe (les haïsseurs des Juifs) à travers des récits criminalisant un autre groupe (les Juifs) qui est, lui, innocent des crimes qu'on lui impute. Selon ce scénario, les victimes imaginaires se substituent aux victimes réelles pour transformer ces dernières en responsables des malheurs réels ou potentiels du genre humain, en leur attribuant des intentions criminelles. Une fois la figure du bourreau imaginaire créée et incrustée dans les représentations collectives, il devient légitime de se venger de lui en l'éliminant.

The analysis of victimhood studied in this text is based on the accusation of ritual murder launched against the Jews. This accusation allows for the construction of Jews haters as victims. Thus, it justifies and legitimizes the measures of expulsion and extermination of the Jews since the $12^{\text {th }}$ century. We analyze here the sources of a victim inversion that has occurred the time of Christian anti-Judaism and which is being constantly performed nowadays in the discourses legitimizing violence against Jews and against the Jewish state. This phenomenon has become a well-oiled mechanism run for centuries; it is currently in use in some media discourses and on social networks. It enacts the self-victimization of a group (the haters of the Jews) through criminalizing narratives of another group (the Jews) which is innocent. In this script, the imaginary victims are substituted to the real victims and makes the latter responsible for the real or potential misfortunes of the world by attributing them criminal intentions. Once the figure of the imaginary executioner is created and embedded in the collective representations, it becomes legitimate to take revenge on him by eliminating him.

\section{INDEX}

Mots-clés : autovictimisation, inversion victimaire, meurtre rituel

Keywords : ritual murder, self-victimization, victim inversion 
AUTEUR

PIERRE-ANDRÉ TAGUIEFF

CNRS ; Centre de recherche politique de Sciences Po (CEVIPOF), Paris 